\title{
LA-UR-16-29023
}

Approved for public release; distribution is unlimited.

Title: $\quad$ Operational Characteristics of an Accelerator Driven Fissile Solution System

Author(s): $\quad$ Kimpland, Robert Herbert

Intended for: Report

Issued:

2016-11-28 
Disclaimer:

Los Alamos National Laboratory, an affirmative action/equal opportunity employer, is operated by the Los Alamos National Security, LLC for the National Nuclear Security Administration of the U.S. Department of Energy under contract DE-AC52-06NA25396. By approving this article, the publisher recognizes that the U.S. Government retains nonexclusive, royalty-free license to publish or reproduce the published form of this contribution, or to allow others to do so, for U.S. Government purposes. Los Alamos National Laboratory requests that the publisher identify this article as work performed under the auspices of the U.S. Department of Energy. Los Alamos National Laboratory strongly supports academic freedom and a researcher's right to publish; as an institution, however, the Laboratory does not endorse the viewpoint of a publication or guarantee its technical correctness. 


\section{Operational Characteristics of an Accelerator Driven Fissile Solution System}

Robert Kimpland

\section{$\underline{\text { Introduction }}$}

Operational characteristics represent the set of responses that a nuclear system exhibits during normal operation. Operators rely on this behavior to assess the status of the system and to predict the consequences of off-normal events. These characteristics largely refer to the relationship between power and system operating conditions. The static and dynamic behavior of a chain-reacting system, operating at sufficient power, is primarily governed by reactivity effects. The science of reactor physics has identified and evaluated a number of such effects, including Doppler broadening and shifts in the thermal neutron spectrum. Often these reactivity effects are quantified in the form of feedback coefficients that serve as coupling coefficients relating the neutron population and the physical mechanisms that drive reactivity effects, such as fissile material temperature and density changes. The operational characteristics of such nuclear systems usually manifest themselves when perturbations between system power (neutron population) and system operating conditions arise. Successful operation of such systems require the establishment of steady equilibrium conditions. However, prior to obtaining the desired equilibrium (steady-state) conditions, an approach from zero-power (startup) must occur. This operational regime may possess certain limiting system conditions that must be maintained to achieve effective startup. Once steady-state is achieved, a key characteristic of this operational regime is the level of stability that the system possesses. Finally, a third operational regime, shutdown, may also possess limiting conditions of operation that must be maintained. This report documents the operational characteristics of a "generic" Accelerator Driven Fissile Solution (ADFS) system during the various operational regimes of startup, steady-state operation, and shutdown. Typical time-dependent behavior for each operational regime will be illustrated, and key system parameters, such as response times, will be quantified. A generalized linear systems analysis of steady-state operations will be performed to evaluate the level of stability of ADFS systems. This information should provide a basic understanding of typical ADFS system operational behavior, and facilitate the development of monitoring procedures and operator aids.

\section{$\underline{\text { Generic ADFS System Model }}$}

A generic ADFS system consists of a homogeneous aqueous fissile solution with a volume large enough to possess a significant multiplication factor $\left(k_{\text {eff }}\right)$. An accelerator provides a source of external neutrons that interact with the fissile solution and cause fission. The behavior of any specific configuration should not differ materially from the generic ADFS system, because the reactivity effects are largely a function of the fissile solution composition, and they are driven by solution temperature and density changes. While certain variations in geometry and material composition may occur, these physical effects are universal to all ADFS systems. The simulation model described in "A Generic System Model for a Fissile Solution Fueled Assembly," LA-UR-13-22033, by Kimpland \& Klein, was used to investigate the operational characteristics of a generic ADFS system. The model focuses on key system components that contribute directly to reactivity effects in the fissile solution. The system model consists of a fissile solution core with its associated cooling structures and a gas plenum.

The generic ADFS system model has a nominal operating power of $60 \mathrm{~kW}$ for a neutron source strength of $4 \times 10^{13} \mathrm{n} / \mathrm{s}$. The thermal-hydraulic characteristics are such that the average operating fuel (fissile 
solution) temperature is $70^{\circ} \mathrm{C}$. The fuel is $20 \%$ enriched uranyl-sulfate with a uranium concentration of $140 \mathrm{gU} /$ liter. The fuel volume is 272 liters. It is believed that these system parameters and operating conditions are representative of any proposed ADFS system, based on current concept designs.

\section{$\underline{\text { Reactivity Feedback }}$}

As noted above, reactivity effects play a central role in the operation of an ADFS system. A Low Enriched Uranium (LEU) homogeneous aqueous fissile solution is affected by several reactivity mechanisms. Doppler broadening in Uraniium-238 resonances and shifts in the thermal neutron spectrum produce significant cross-section changes. Temperature driven fuel solution expansion leads to enhanced neutron leakage, by decreasing solution density and by altering the solution geometry. These effects are typically combined into a single temperature coefficient of reactivity. The generic ADFS system model uses an average temperature coefficient of $-0.037 \$ /{ }^{\circ} \mathrm{C}$, which is consistent with measured values from fissile solution fueled research reactors. In addition to these reactivity effects, fissile solutions are affected by void formation. Radiolytic gas voids reduce the density of the fuel and alter the solution geometry by displacing fuel. These effects are often quantified in a void coefficient of reactivity. The generic ADFS system model uses an average value of $-105 \$ / \mathrm{m}^{3}$, which represents the reactivity change as a function of gas volume. These two reactivity coefficients are the dominant reactivity feedbacks common to all ADFS systems. However, several other system specific reactivity feedbacks may also be significant, such as temperature feedback coefficients for structural components, coolant regions, and neutron reflectors.

\section{Radiolytic Gas}

The generation of radiolytic gases in aqueous fissile solutions necessitates the use of a gas plenum above the fuel solution, and an off-gas handling system to continuously sweep the plenum. As noted in the previous section, radiolytic gas has a significant effect on reactivity feedback. However, radiolytic gas affects other system parameters that may impact operating conditions. Gas voids in the fuel solution will affect solution flow patterns, which affect heat transfer from the fuel solution to the coolant. Gas eventually flows through the fuel solution and exits into the plenum. Perturbations in gas production may affect the performance of the off-gas handling system by altering system pressure. Also, the interface between the fuel solution and the plenum may develop a foam structure due to the entrainment of solution. These effects are simply noted here, as operational considerations. Similar behaviors have been observed in solution fueled reactors.

\section{$\underline{\text { Startup }}$}

The startup process begins with a zero-power operation in which fuel solution is introduced into the target vessel. This operation will take the form of an "approach to critical," due to the significant multiplication factor of proposed ADFS concepts. Once the fuel has been loaded into the target vessel and the auxiliary systems have achieved the appropriate level of readiness, powered operation can begin.

At this point, certain assumptions about the operating conditions must be made. First, the fuel is in its maximum reactivity configuration and the fuel temperature is in equilibrium with the coolant inlet temperature. Second, the external neutron source (accelerator) is initiated as a step insertion. Clearly, a wide variety of powered startup paths may be envisioned. For example, the external neutron source may possess the capability of initiating with a ramp insertion. Also, the fuel may be externally preheated 
prior to the external neutron source initiation. However, the powered startup conditions listed above are operationally the simplest, but would also produce the most dynamic behavior possible. Thus, this startup scenario will reveal the most extreme operating conditions during normal operation.

The system model was used to simulate the startup of the generic ADFS system, given the system parameters and operating conditions stated above. Figure 1 shows the behavior of the system immediately following the initiation of the external neutron source. System parameters are presented in normalized units to illustrate their relative behavior. The initial fuel temperature was assumed to be $20^{\circ} \mathrm{C}$, and the initial system reactivity was $-0.40 \$$. The startup begins with a power pulse, characterized by a sharp rise in power followed by a slower decline in power and a broad tail. The power reaches a maximum of $200 \mathrm{~kW}$, then falls to $83 \mathrm{~kW}$ at 400 seconds. The fuel temperature increases steadily to $57^{\circ} \mathrm{C}$ at 400 seconds, while the system reactivity shows a corresponding drop to $-1.75 \$$. This system response is caused by external neutrons being rapidly multiplied and power growing quickly. However, once the system's thermal inertia is overcome, the fuel temperature begins to increase, it then pushes the system reactivity lower through the temperature coefficient of reactivity and neutron multiplication decreases. While the initial rise in system power occurs on a time scale that may challenge potential operator responses, the fuel temperature rise occurs on a time scale amenable to operator response, if so desired. 


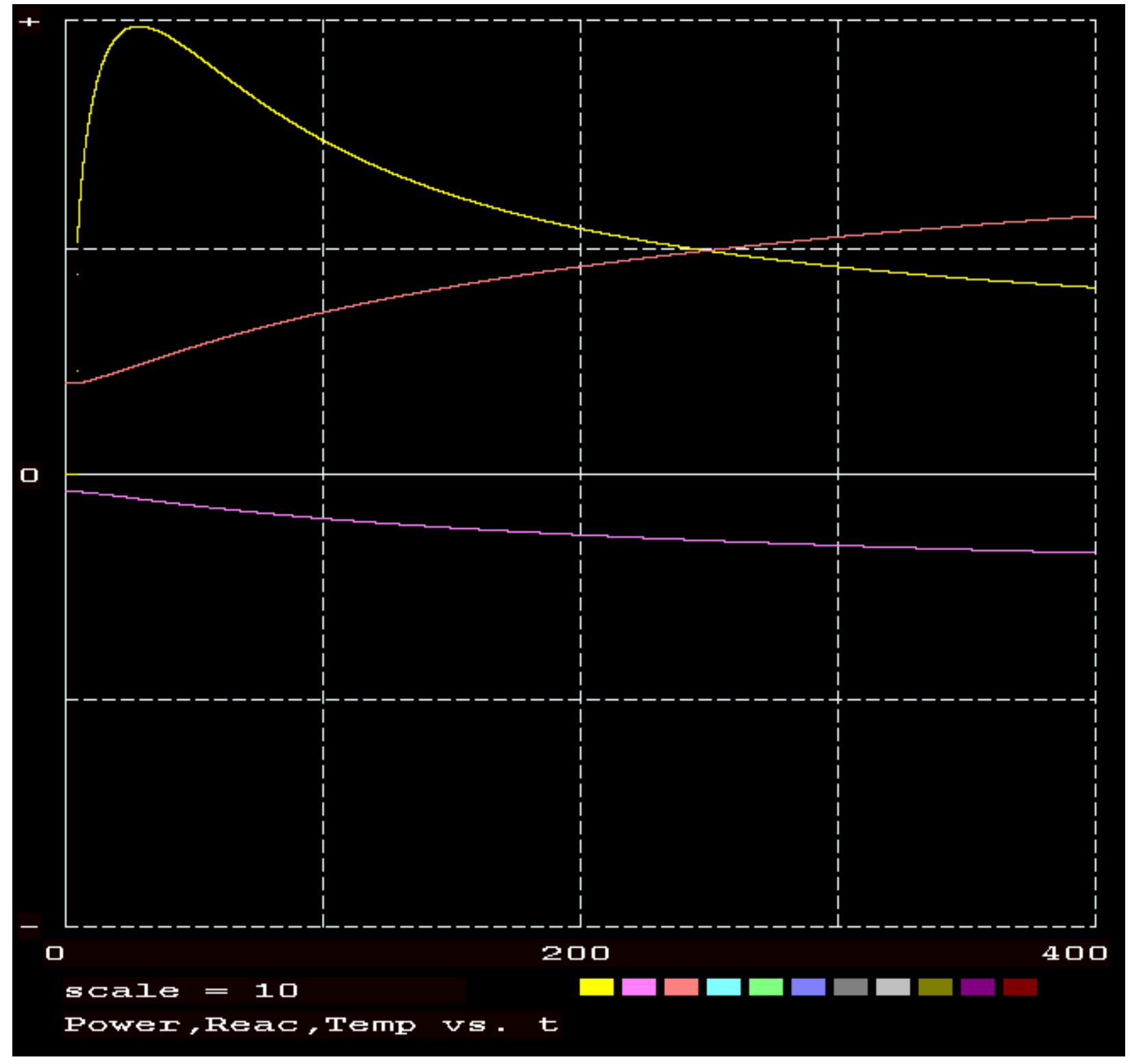

Figure 1. Model's simulation of startup over the first 400 seconds of the transient; Power= average system power, Reac= system reactivity, and Temp= average fuel temperature.

The unmitigated power pulse at the beginning of the startup process may produce unfavorable operating conditions, if the initial fuel solution configuration produces an undesirable initial reactivity. The system model was used to determine the peak power as a function of the initial system reactivity. Table 1 summarizes these results. The peak power is relatively sensitive to the initial system reactivity, which points out the need for a certain level of precision in the zero-power approach operation. This relationship may serve as an operational indicator or confirmation of the status of the system prior to initiation of the external neutron source. 
Table 1. Peak power as a function of initial reactivity.

\begin{tabular}{|l|l|}
\hline Initial reactivity $(\$)$ & Peak power $(\mathrm{kW})$ \\
\hline-0.05 & 321 \\
\hline-0.10 & 295 \\
\hline-0.20 & 255 \\
\hline-0.40 & 200 \\
\hline-0.80 & 135 \\
\hline
\end{tabular}

An important effect of fission energy deposition in the fuel solution is the onset of radiolytic gas production. Dissolved Hydrogen and Oxygen gas, produced by radiolysis, builds during startup. Once a threshold concentration of dissolved gas is reached, the gas is released from the fuel solution in the form of voids. The threshold concentration of each gas and its variation with solution operating conditions are not well known. Following the threshold point, void production is assumed to be directly proportional to power, because it is believed that fission tracks created in the solution serve as nucleation sites for bubble formation. The startup process cannot be considered complete until the threshold point is reached and the system is allowed to adjust to the reactivity effects of voids in the fuel solution. Figure 2 shows the effects of radiolytic gas on startup. The gas void fraction curve shows the Hydrogen gas coming out of solution first, followed by the Oxygen. The presence of gas voids in the fuel solution cause an increase in negative reactivity through the void coefficient of reactivity, which in turn pushes the neutron multiplication down further. The gas thresholds were chosen arbitrarily, but it is believed that Hydrogen will appear first, because it is produced directly from radiolysis, while Oxygen is formed from secondary reactions. It is important to note that the rate of void formation is directly proportional to power, so if threshold occurs when the power is much larger, then the rate of void formation would be much larger. This could affect the operation of the plenum and the off-gas handling system, which could in turn lead to reactivity effects. Also, the trajectory of the power curve in time would be altered by perturbations in the void reactivity feedback, which could serve as an indication of anomalous conditions from one startup to the next. It should further be noted that the onset of radiolytic gas production may occur on a time scale too fast for any potential operator response. 


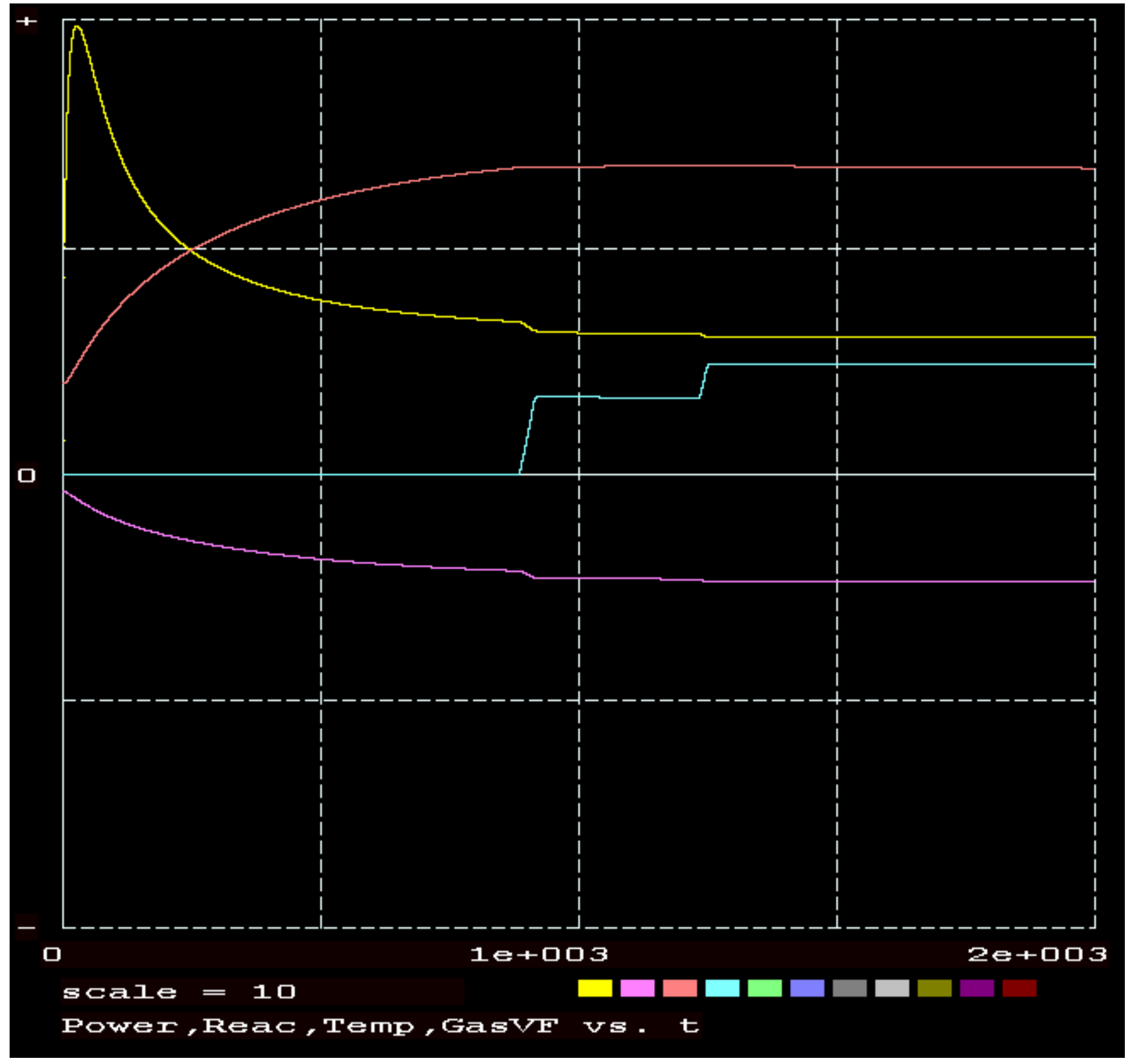

Figure 2. Model's simulation of startup over the first 2000 seconds of the transient; Power= average system power, Reac= system reactivity, Temp= average fuel temperature, and GasVF= radiolytic gas void fraction in the fuel.

After the generation of void in the fuel begins, the system will slowly approach equilibrium conditions by balancing the parallel reactivity feedbacks of fuel temperature and void fraction in the fuel. The fuel temperature reflects a balance between the power and the heat removal system. The characteristic time constant for this process is a function of the fuel solution thermal inertia and the overall heat transfer coefficient. The void fraction reflects a balance between the power and the transport of void out of the fuel solution. The characteristic time constant of this mechanism is a function of the bubble residence time. Around 2000 seconds the simulation in Figure 2, reaches equilibrium. The equilibrium power is $63 \mathrm{~kW}$, the average fuel temperature is $70^{\circ} \mathrm{C}$, and the average void fraction is $0.9 \%$. Radiolytic gas is exiting the fuel solution and entering the plenum at a rate of 28 liters per minute. The system reactivity has dropped to $-2.28 \$$, with $88 \%$ of the reactivity loss due to fuel temperature effects and 
$12 \%$ due to void effects. Once equilibrium conditions have been reached, startup ends and the steadystate operating regime begins.

The at-power startup operating regime may be characterized as an extended transient with a duration of several tens of minutes. During this period, the system power works to overcome fuel solution thermal inertia, while continuously adjusting to temperature and void reactivity feedback. Radiolytic gas appears and interacts with the plenum and off-gas handling system.

\section{$\underline{\text { Steady-State Operations }}$}

It is anticipated that an ADFS system will operate with very little direct input from operators during steady-state operations. However, system monitoring will increase, because a number of processes must occur to maintain power. For example, water losses due to radiolysis must be replenished and, depending on the type of fuel solution, certain chemical balances, such as $\mathrm{pH}$, must be adjusted as energy is deposited into the fuel solution. These processes may be continuous, periodic, or on demand. Also, the heat removal system must maintain a constant fuel solution temperature and the off-gas handling system must maintain a constant pressure in the plenum to avoid power fluctuations.

A typical ADFS system will require a number of auxiliary support systems to maintain steady operation at power. Chief among these systems is a heat removal system, which includes fuel solution coolant structures, piping, pumps, and an ultimate heat sink. An off-gas handling system, which will include a number of components to sweep and condition the plenum cover gas. And, a water makeup system, which may include additional components for fuel solution chemical control. These systems interact directly with the fuel solution and may create perturbations in the operating conditions, which will create perturbations in system reactivity. The extent to which an ADRS system can maintain equilibrium, over the range of all operating conditions, would require a detailed knowledge of all the systems that interact with the fuel. However, we may quantify some key operational characteristics with the generic system model, using transient response and linear systems techniques.

We begin with the time-dependent system model, by simulating the transient response to a simple reactivity perturbation. From equilibrium, a step insertion of reactivity is introduced. Figure 3 shows the model's simulation of a 0.5 insertion of reactivity occurring at 2000 seconds (equilibrium). The power curve shows a prompt jump in power following the step insertion of reactivity. The gas void fraction follows the power, while the fuel temperature rises gradually. The characteristic time constant of the radiolytic gas void fraction is clearly much smaller than that of the fuel temperature. Thus the void reactivity feedback reacts on a time scale much faster than that of the temperature feedback. The system reacquires equilibrium conditions quickly, via a minimal power trajectory. Oscillatory behavior appears to be nonexistent or totally damped down. A number of simulations were run with various sized step insertions, but the same behavior was observed in all cases. The system reacts smoothly to these reactivity changes, re-establishing equilibrium conditions with a minimum number of power fluctuations. 


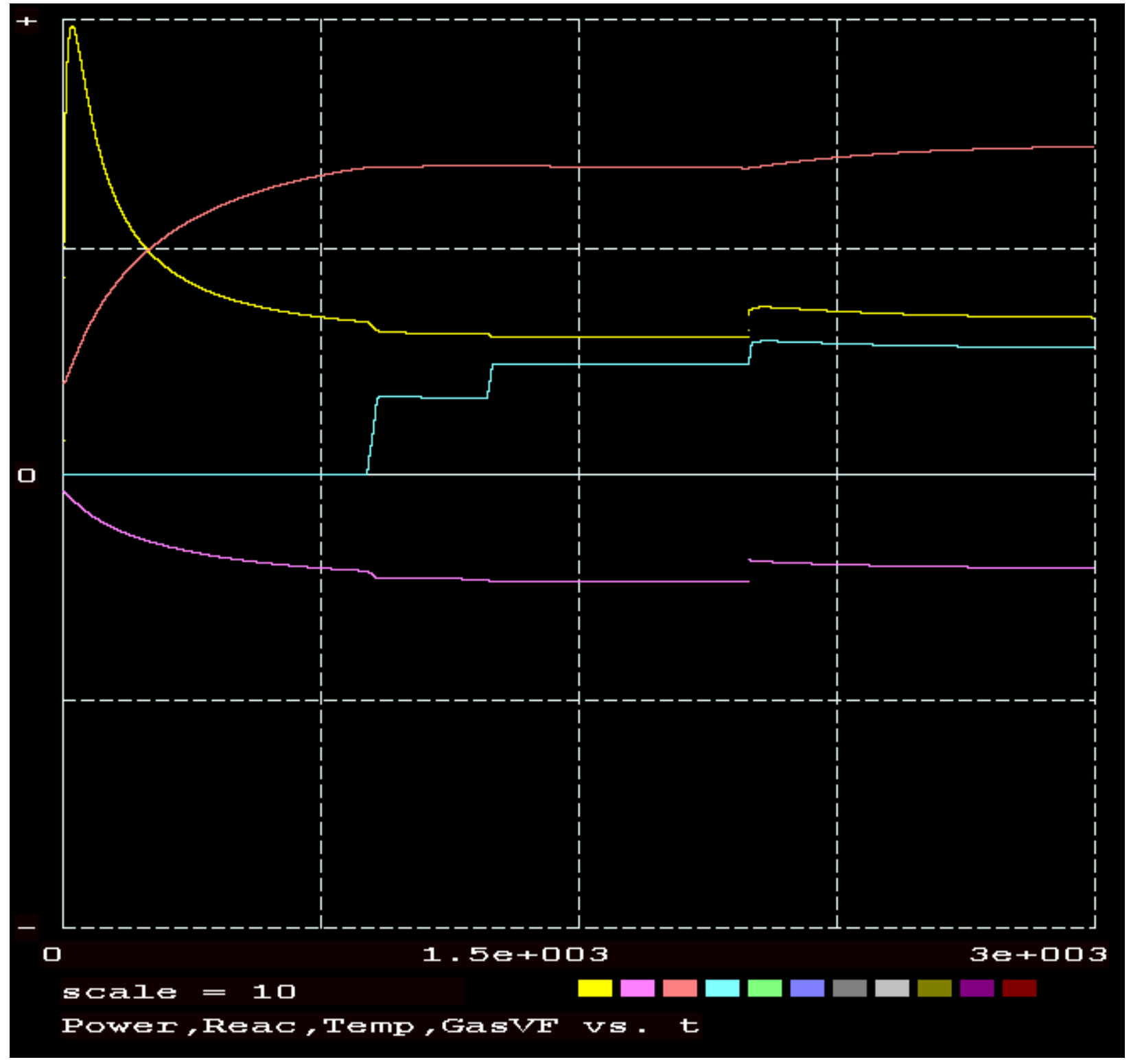

Figure 3. Model's simulation of $0.5 \$$ step insertion at 2000 seconds; Power= average system power, Reac $=$ system reactivity, $T e m p=$ average fuel temperature, and GasVF= radiolytic gas void fraction in the fuel.

The results illustrated in Figure 3 clearly show that system power does not diverge, given a positive insertion of reactivity during equilibrium conditions. This behavior is expected, given that the reactivity feedback coefficients are both negative. Negative feedback occurs when some function of system output, in this case power, is fed back into the system, through reactivity feedback coefficients, in a manner that tends to reduce fluctuations in the output. In general, negative feedback tends to promote a settling to equilibrium, and reduces the effects of perturbations. However, even though negative feedback ensures non-divergent behavior, it is not sufficient to ensure stability. Since an ADFS system is a chain-reacting system, albeit subcritical, we shall apply the criterion of reactor stability. Reactor stability states that a system initially in equilibrium, re-establishes equilibrium conditions following a reactivity perturbation. The techniques developed in "Stability of Fissile Solution Systems," Los Alamos 
National Laboratory Document LA-UR-15-22590, by Kimpland and Klein, are used here to assess the stability of the generic ADFS system model. This document will be referred to as "SFSS" below.

While time-dependent simulations provide valuable information on how the system responds to certain inputs, they reveal little information on why the system responds the way it does. For this level of analysis we turn to frequency response techniques. Frequency response is the quantitative measure of the output spectrum of a system in response to a stimulus, and is used to characterize the dynamics of the system. It is a measure of magnitude and phase angle of the output as a function of frequency, in comparison to the input. In simplest terms, if a sine wave is injected into a system at a given frequency, a linear system will respond at that same frequency with a certain magnitude and a certain phase angle relative to the input.

Transfer functions for both a fissile solutiOon reactor and an ADFS system are described in SFSS. The forward transfer function ("zero-power" or "zero-feedback" transfer function), relating changes in power to changes in reactivity, in the frequency domain, is given by

$$
G_{1}=\frac{\delta N}{\delta R}=\frac{n_{o}}{\left[\frac{\Lambda}{\beta} s-r_{o}+\sum_{k=1}^{m} F_{k} \frac{s}{s+\lambda_{k}}\right]} .
$$

For the generic ADFS system, we include two simplified reactivity feedback loops, a temperature feedback loop given by

$$
H_{1}=\frac{\alpha_{T} K_{f}}{s+g_{1 f}}
$$

and a void feedback loop given by

$$
H_{2}=\frac{\varphi G_{v}}{s+g_{1 v}}
$$

where

$$
K_{f}=\frac{1}{M_{f} C_{p f}} \quad g_{1 f}=\frac{\gamma_{f}}{M_{f} C_{p f}}
$$

$M_{\mathrm{f}}$ is the total fuel mass, $\gamma_{f}$ the overall heat transfer coefficient from the fuel to the coolant, $C_{p f}$ is the specific heat of the fuel, $G_{v}$ is the radiolytic gas production rate, and $g_{1 v}$ is the inverse gas bubble residence time in the fuel. These equations satisfy the reactivity feedback model given by

$$
\delta R=\alpha_{T} \delta T_{f}+\varphi \delta V
$$


which relates the change in system reactivity to changes in fuel temperature and void volume, through reactivity feedback coefficients. The closed-loop transfer function for this system of equations is given by

$$
G_{2}=\frac{G_{1}}{1+G_{1}\left(H_{1}+H_{2}\right)}
$$

and the open-loop transfer function is given by

$$
G_{3}=G_{1}\left(H_{1}+H_{2}\right) \text {. }
$$

The term "stability" is often used very generally to describe various aspects of system dynamics. Asymptotic stability refers to an absolute measure of system stability in the linear approximation, given a specific set of system parameters. Robustness is a measure of how far from instability a system operates. Within the context of frequency response analyses, the terms "more stable" or "less stable" refer to the robustness of the system, which may be quantified in various ways. Finally, frequency response analysis may reveal important characteristics of system dynamics in the time domain, such as regenerative (reinforcing) behavior. This behavior usually manifests itself as damped oscillations during time-dependent responses. Reinforcing behavior occurs when the open-loop phase shift lags enough to strengthen the input rather than weaken it. In this time domain context, "more stable" usually refers to the absence of such behavior.

In SFSS, it was shown that fissile solution systems, possessing physical properties consistent with the generic ADFS system model, were asymptotically stable. The poles of their closed-loop transfer functions were real and negative. We can quickly confirm this result for the generic ADFS system model by examining its open-loop Nyquist plot. The Nyquist plot is a conformal mapping of the open-loop transfer function in the complex plane. It is used to assess the stability of systems with negative feedback. The real part of the open-loop transfer function is plotted on the X-axis and the imaginary part is plotted on the $\mathrm{Y}$-axis. Frequency is tracked along a closed contour as a parameter, resulting in a plot of the open-loop frequency response. Figure 4 shows the Nyquist plot for the generic ADFS system model. A visual inspection of the poles of the open-loop transfer function will reveal that they are all real and negative. Note that the denominator of the forward transfer function is simply the inhour equation, whose roots are real and negative for subcritical systems. Therefore, if the Nyquist plot does not encircle the critical point $(-1+0 j)$, then the poles of the closed-loop transfer function are real and negative.

Clearly, the Nyquist plot does not encircle the critical point, which means that the generic ADFS system model is asymptotically stable in the linear approximation. 


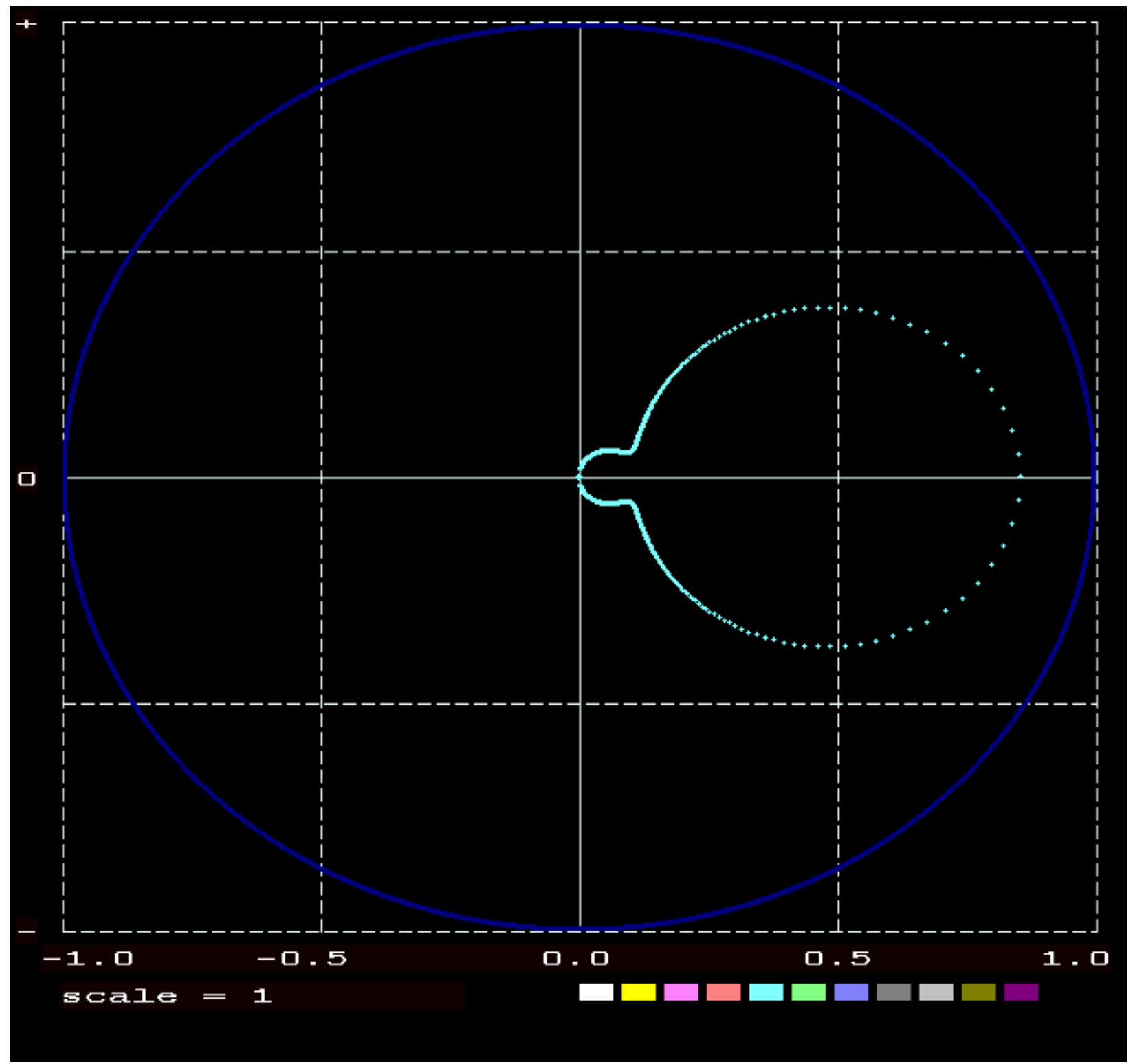

Figure 4. Nyquist plot for the generic ADFS system model open-loop transfer function $\left(\mathrm{G}_{3}\right)$ with a steadystate power of $63 \mathrm{~kW}$ and a reactivity of -2.28 ; green $=G_{3}$, gray= unit circle.

Also, included in Figure 4 is the unit circle, which denotes an amplitude of unity. Typically, a quantitative measure of robustness, which is a measure of how far from the threshold of instability the system operates, would be illustrated by the phase margin. The threshold of instability is the area surrounding the critical point. However, the Nyquist plot for the ADFS system model open-loop transfer function never crosses the unit circle, which means the amplitude of the open-loop transfer function never exceeds unity and the phase margin is not quantifiable. However, we may note that the path of the open-loop transfer function mostly lies in the positive real half of the complex plane, and never even remotely approaches the critical point. This indicates that the system normally operates far from the onset of instability.

To evaluate the frequency response of the closed-loop transfer function $\left(\mathrm{G}_{2}\right)$, we use Bode plot representations. Bode plots are a combination of a Bode magnitude plot, which tracks the logarithm of 
the transfer function's amplitude, and a Bode phase angle plot, which tracks the phase shift of the transfer function. Both plots are usually plotted against the logarithm of the angular frequency. In addition to explicit quantitative results, Bode plots provide qualitative information about system dynamics. Some characteristics of time-dependent behavior may be inferred from the shape of Bode plots.

Figure 5 shows the Bode magnitude plot for the generic ADFS system. Both the closed-loop and forward transfer functions are shown over the angular frequency range from $10^{-4}$ to 10 radians $/ \mathrm{s}$. The closedloop begins to diverge from the forward transfer function between 1 and 10 radians/s, indicating that the system cannot react fast enough for any significant feedback to manifest itself for sufficiently fast transients. Above 10 radians/s the amplitude of both transfer functions drop to insignificant levels, and we ignore that part of the frequency range from here on. The closed-loop response is characterized by a broad gentle slope. No sharp resonances occur and the closed-loop transfer function never crosses the forward transfer function, indicating that no reinforcing behavior occurs. This means that oscillatory behavior is unlikely to occur in the time domain, which was demonstrated in Figure 3. Similar results were obtained for different steady-state power levels. In each case, no resonances were observed, confirming the results of the Nyquist plot that the system never approaches the threshold of instability. 


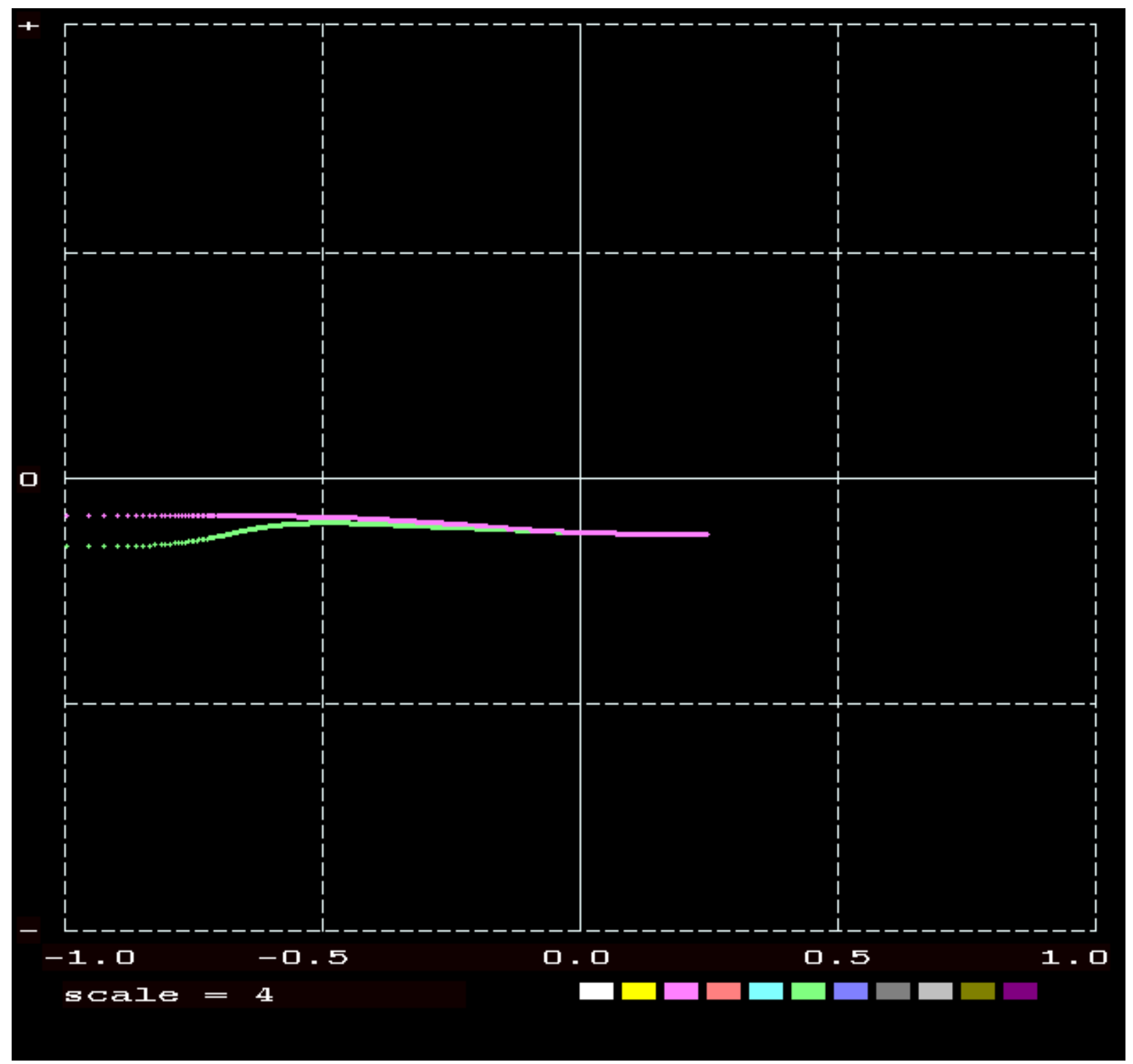

Figure 5. Bode magnitude plot for the ADFS system model closed-loop transfer function over the angular frequency range $10^{-4}$ to 10 radians $/ \mathrm{s}$; purple $=\log \left(\operatorname{Cabs}\left(G_{1}\right)\right)$, green $=\log \left(\operatorname{Cabs}\left(G_{2}\right)\right)$.

Figure 6 shows the Bode phase angle plot for the closed-loop transfer function. This curve shows that for sufficiently slow transients the system reacts fast enough to lead reactivity changes. While, for faster transients, the system reacts slowly and lags reactivity changes. 


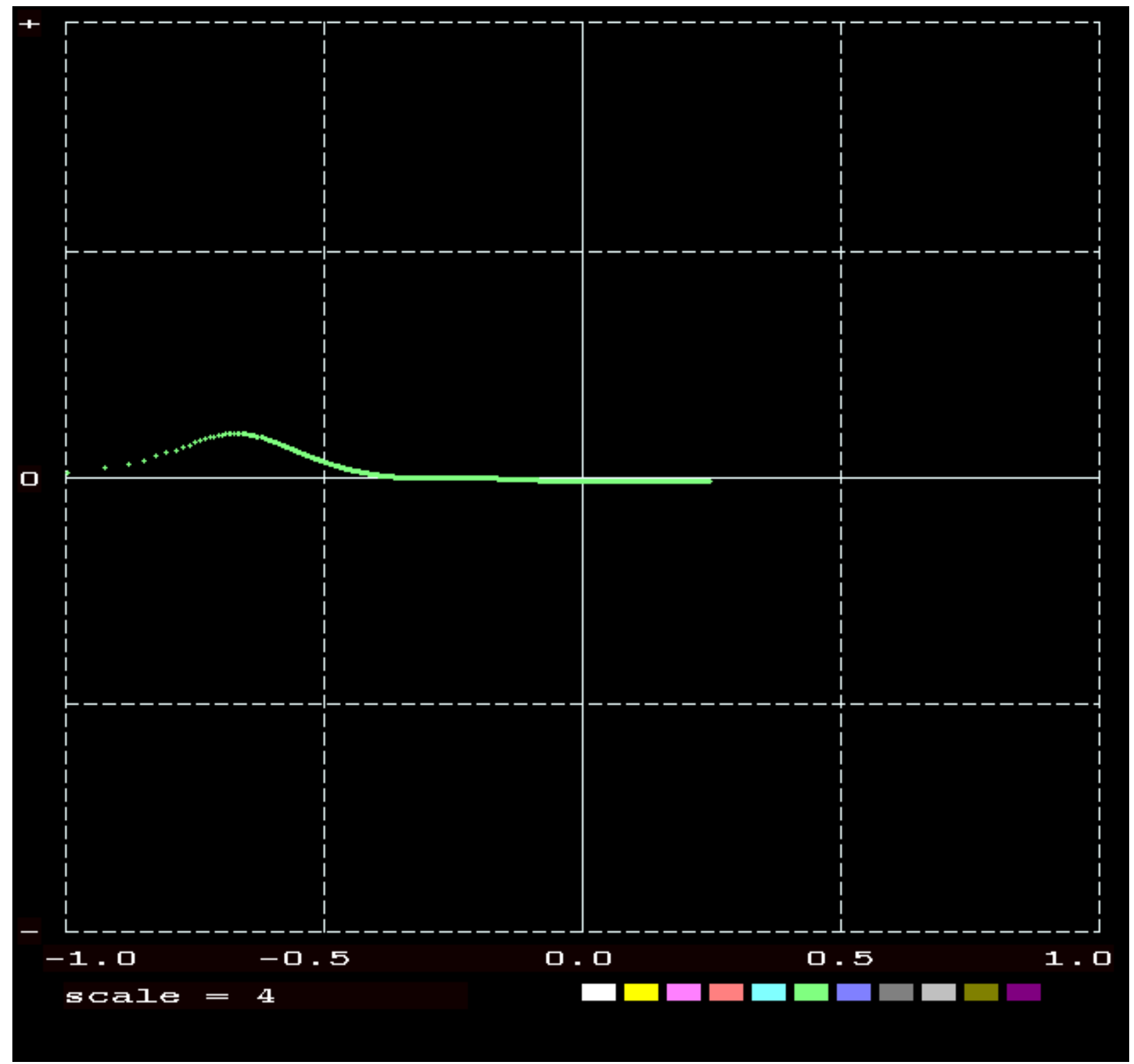

Figure 6. Bode phase angle plot for the ADFS system model closed-loop transfer function, $\operatorname{Arg}\left(G_{2}\right)$ in radians, over the angular frequency range $10^{-4}$ to 10 radians/s.

Both the open-loop and closed-loop frequency response show a high degree of system stability. A physical explanation for this behavior may be inferred from the frequency response of individual components in the open-loop transfer function. Figure 7 shows the Bode magnitude plot of the forward transfer function at $-2.28 \$$ and at $0.0 \$$ (critical). The forward transfer function represents the neutron kinetics, relating system power to input changes in reactivity, in the absence of feedback. The curves show a significant suppression of response amplitude with level of sub-criticality. As a result, the amplitude of the open-loop transfer function is significantly reduced. Typically, a feedback system will become more stable when the open-loop amplitude is reduced, because reinforcing behavior is minimized by less amplification of feedback. 


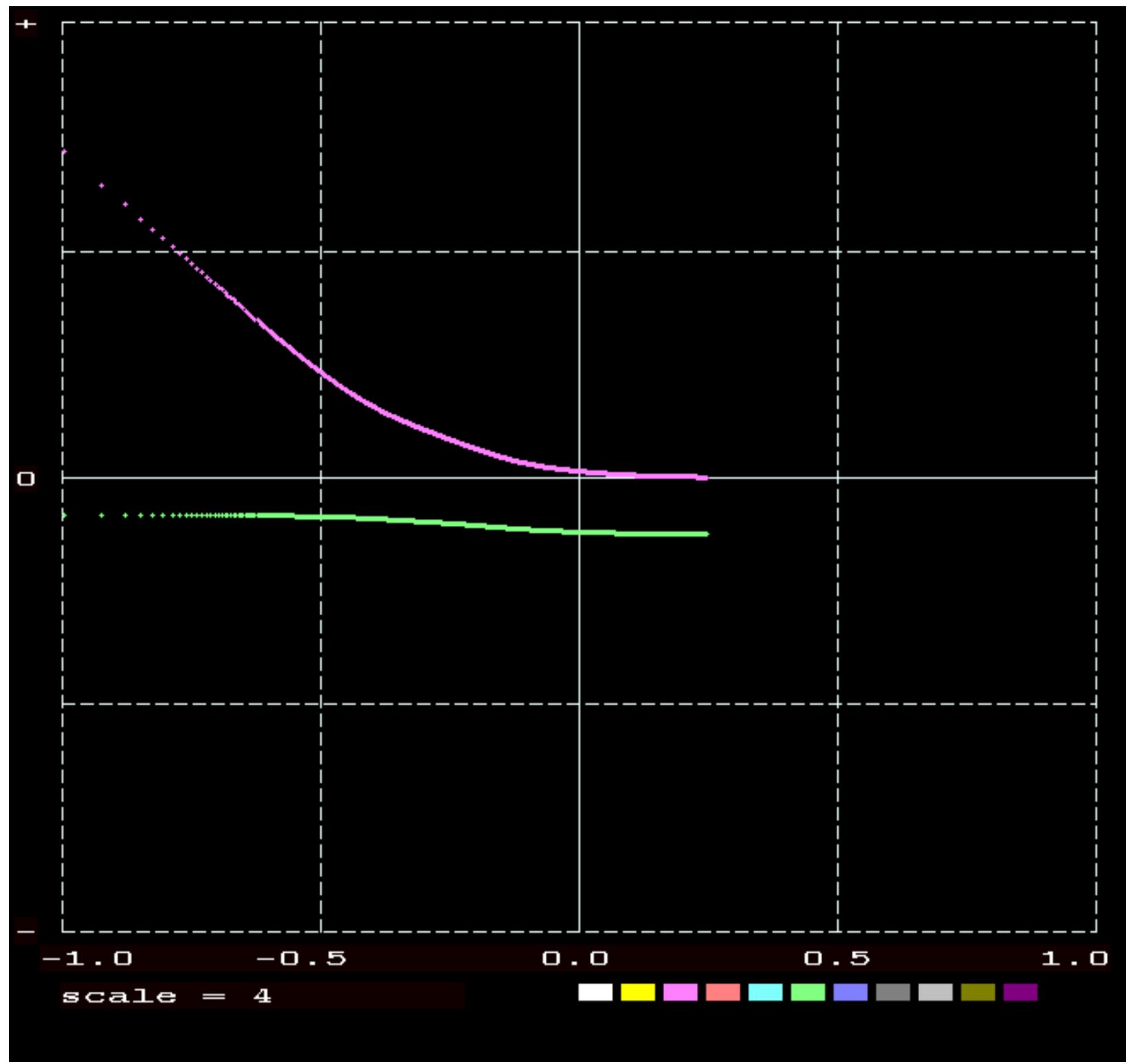

Figure 7. Bode magnitude plots for the forward transfer function, $\log \left(\operatorname{Cabs}\left(\mathrm{G}_{1}\right)\right)$, over the angular frequency range $10^{-4}$ to 10 radians/s; green $=-2.28 \$$, purple $=0.0 \$$ (critical).

Figure 8 shows the Bode magnitude plots for both the temperature and the void reactivity feedback transfer functions, $\mathrm{H}_{1}$ and $\mathrm{H}_{2}$ respectively. The temperature feedback dominates for very slow transients, then drops off quickly for faster transients. The void feedback dominates for faster transients, then drops for very fast transients. The crossover point occurs around 0.01 radians/s, which reflects the characteristic time constants for fuel temperature and void fraction change, represented by the inverse of $g_{1 f}$ and $g_{1 v}$, respectively. The generic ADFS system model has a fuel temperature characteristic time constant of 704 seconds, while the void fraction characteristic time constant is 5 seconds. The significance of these values is that when the temperature feedback drops off, because it can't keep up with faster transients, the void feedback remains elevated. The combination of the two parallel feedbacks, with distinct time constants, cover nearly the entire range of frequencies. The void feedback begins to drop off around 1 radian/s, however, at this point the amplitude of the forward and closed-loop transfer functions begin to merge. 


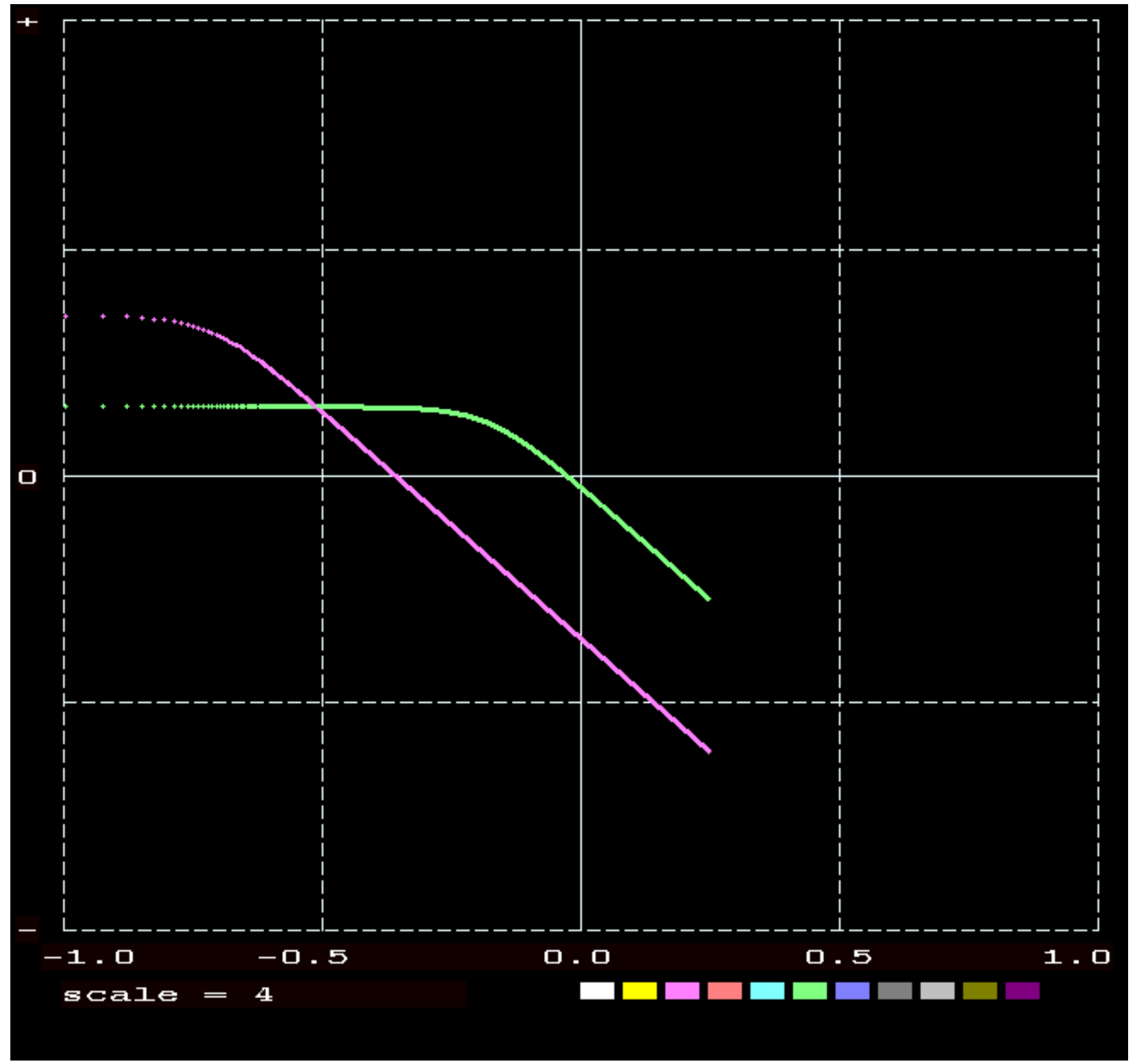

Figure 8. Bode magnitude plots for both temperature and void reactivity feedback over the angular frequency range $10^{-4}$ to 10 radians/s; green $=\log \left(\mathrm{Cabs}\left(\mathrm{H}_{2}\right)\right)$, purple $=\log \left(\mathrm{Cabs}\left(\mathrm{H}_{1}\right)\right)$.

Figure 9 shows the Bode phase angle plots for both the temperature and void reactivity feedback. The temperature reactivity feedback lag increases steadily over most of the frequency range, while the void reactivity feedback lag begins its increase after 0.01 radians/s, again reflecting the characteristic time constants. Both reactivity feedbacks approach an asymptotic phase shift value of approximately $-89^{\circ}$. Both reactivity feedback mechanisms react well within a fraction of a half cycle, thus no reinforcing behavior occurs due to a lag in feedback. 


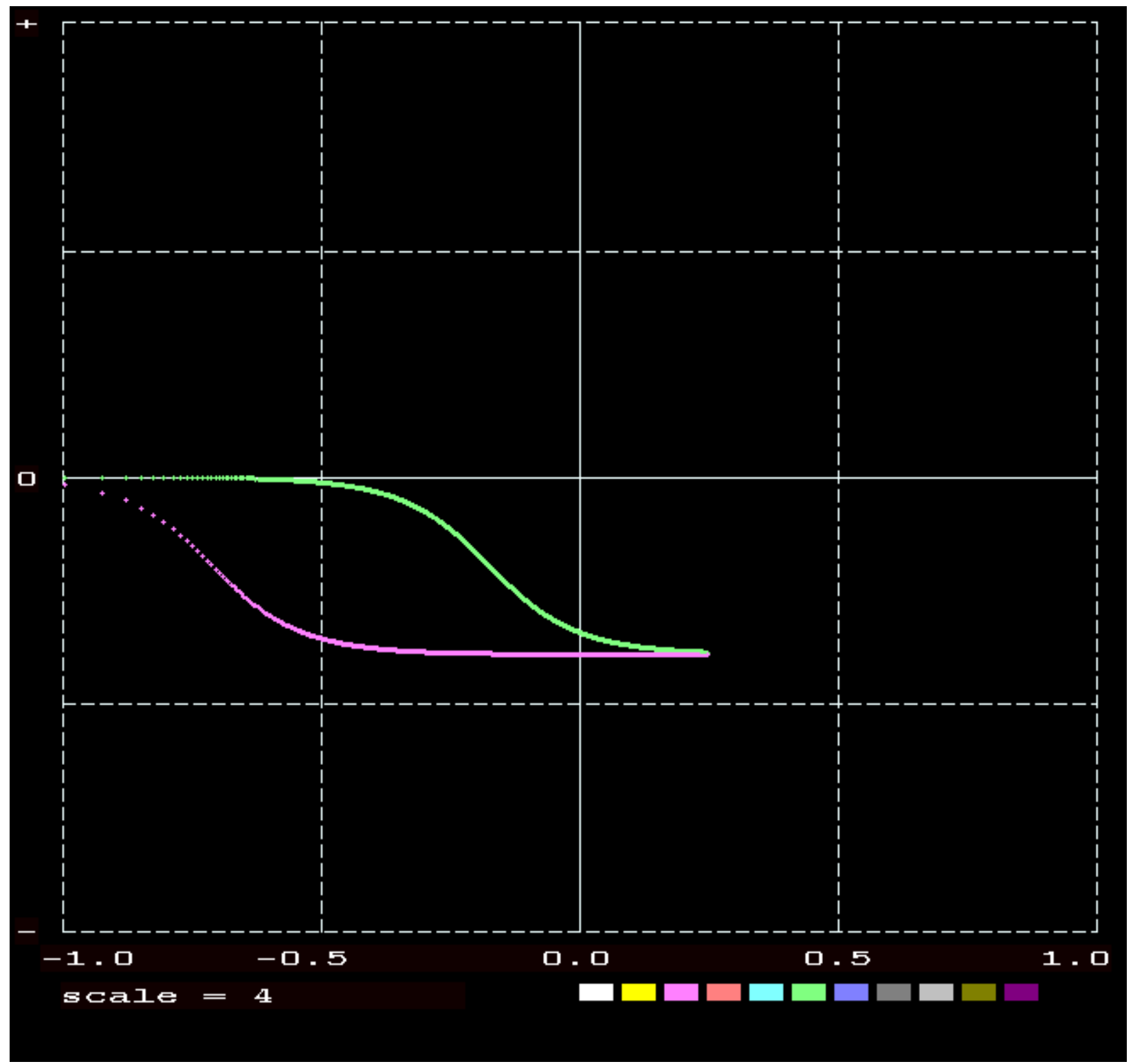

Figure 9. Bode phase angle plots for both temperature and void reactivity feedback, in radians, over the angular frequency range $10^{-4}$ to 10 radians/s; green $=\operatorname{Arg}\left(\mathrm{H}_{2}\right)$, purple $=\operatorname{Arg}\left(\mathrm{H}_{1}\right)$.

Finally, Figure 10 shows both the Bode magnitude and phase angle plots for the ADFS system model open-loop transfer function. As was noted in the Nyquist plot of Figure 4, the amplitude of the openloop transfer function never exceeds unity, while the phase shift never exceeds $-90^{\circ}$. The combination of sub-criticality suppressing the amplitude of the forward transfer function and the two parallel reactivity feedbacks, with distinct time constants, over the frequency range of interest, produce a high level of system stability. With a phase shift that never exceeds $-90^{\circ}$, no reinforcing behavior is possible. 


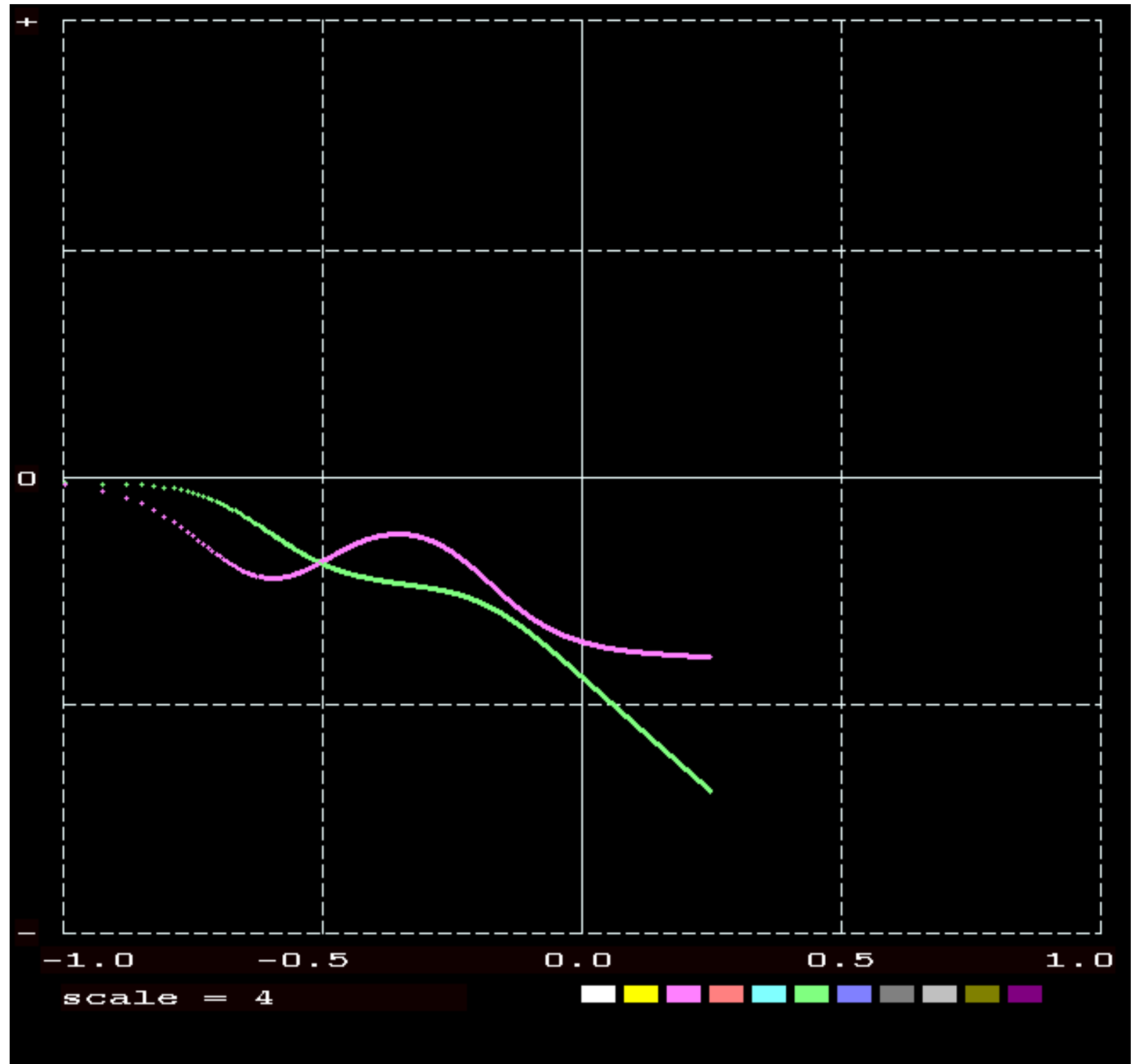

Figure 10. Bode magnitude and phase angle plots for the ADFS system model open-loop transfer function over the angular frequency range $10^{-4}$ to 10 radians/s; green $=\log \left(\operatorname{Cabs}\left(G_{3}\right)\right)$, purple $=\operatorname{Arg}\left(G_{3}\right)$ in radians.

Steady-state operations will largely consist of maintaining nominal full-power conditions. This will entail balancing the requirements of the fuel solution with the function of the auxiliary support systems. Due to the complexity of the overall system, reactivity perturbations may be numerous and routine. However, the preceding system analysis suggests that the ADFS system can adjust to perturbations by quickly settling to equilibrium without oscillatory behavior. Also, the system appears to possess a significant margin between its normal operating conditions and the onset of instability. The two parallel reactivity feedback mechanisms are of paramount importance to this behavior. In particular, the void reactivity feedback due to its short characteristic time constant. However, unlike the temperature coefficient of reactivity, the void coefficient of reactivity may be affected by changes in system operating conditions, such as plenum pressure and solution chemistry. Careful monitoring of system parameters is required to ensure steady operation. 


\section{Shutdown}

Planned shutdown may consists of any number of actions, depending on the specific ADFS system concept design. It may consist of simply turning the external neutron source off and maintaining the fuel solution in the target vessel. Alternatively, it may consist of a sequence of actions, such as performing fuel solution conditioning in the target vessel followed by a fuel dump to an auxiliary fuel solution storage reservoir. For this cursory overview of system operational characteristics, we only consider the most basic action of turning the external neutron source off and maintaining the fuel solution in the target vessel. The goal here is to illustrate the time-dependent response of the system, given unaltered steady operation of the heat removal and off-gas handling systems. Since "powered operations" and their associated reactivity effects are the focus of this report, we neglect long term actions, such as decay heat removal and fuel solution transfers. Figure 11 shows the ADFS system model's timedependent response to the external neutron source being shut off during full-power steady-state operation. The power drops nearly instantaneously to a delayed-neutron tail, where it decays to an effectively "zero-power" condition. The radiolytic gas void fraction follows the power behavior due to its short characteristic time constant. This causes radiolytic gas to disappear from the fuel solution rapidly. As a result, the reactivity feedback created by the radiolytic gas void disappears rapidly. The fuel temperature decays to its initial starting point much more slowly due to its longer characteristic time constant. Thus, the reactivity feedback due to fuel temperature decays slowly. After approximately 2500 seconds from extinguishing the external neutron source, the system re-establishes its pre-startup configuration. 


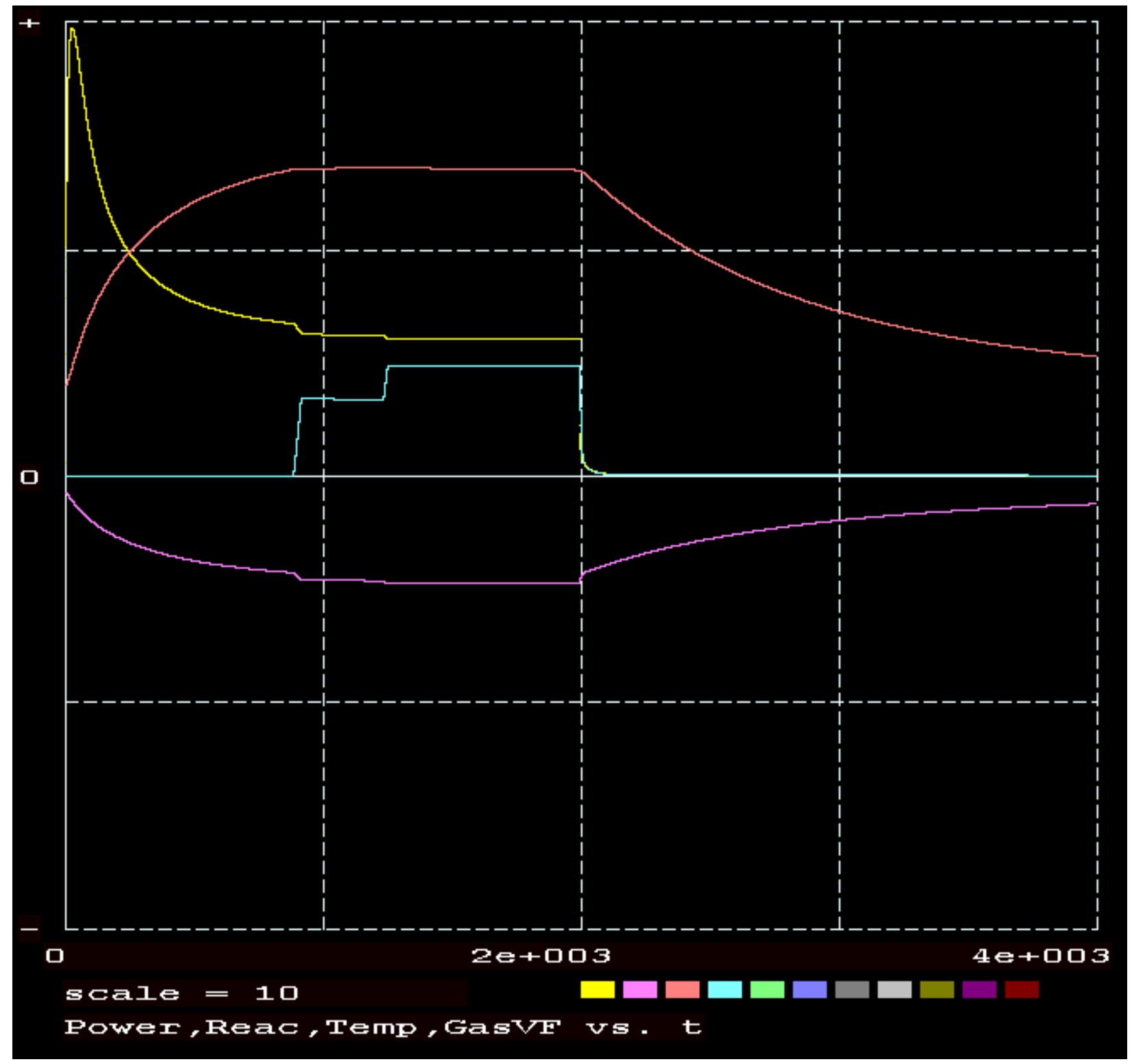

Figure 11. ADFS system model's time-dependent response to the extinguishing of the external neutron source at $t=2000$ seconds; Power= average system power, Reac= system reactivity, Temp= average fuel temperature, and GasVF= radiolytic gas void fraction in the fuel.

The results presented in Figure 11 represent ideal conditions, where no external reactivity perturbations were introduced into the system during steady-state operations. In the absence of these reactivity perturbations, the system will always return to its initial starting conditions, all things being equal. Operationally, allowing the system to return to its initial pre-start configuration may not be desirable, particularly for the final planned shutdown. Changes to long term heat removal or fuel transfer may be implemented to increase the level of sub-criticality. However, due to the large thermal inertia of the fuel solution, the results in Figure 11 show that adequate time exists during shutdown to implement a variety of ultimate shutdown strategies. 


\section{$\underline{\text { Summary and Conclusion }}$}

This report illustrates the operational characteristics of an ADRS system, during its startup, steady-state operation, and shutdown, with time-dependent responses and frequency response spectra. Information on system operating conditions, response times, and system stability is presented. A physical explanation for inherent system stability is offered based on the thermo-physical response of the fuel solution to fission energy deposition.

It has been noted from decades of operational experience that fissile solution fueled reactors, operationally, possess docile behavior. Accelerator Driven Fissile Solution systems possess the same characteristics as there reactor counterparts, two parallel reactivity feedback mechanisms and large fuel solution thermal inertia, relative to system power. In addition, the level of sub-criticality that ADFS systems possess further dampens time-dependent responses.

These operational characteristics suggest a reasonable level of system operability. The ADFS system startup transient is reminiscent of research reactor behavior, such as the Silene "free-run" experiments. While the system power is dynamic in its range, the thermo-physical response of the fuel solution is mild. System response times, generally, do not appear to challenge potential operator responses. However, the onset of radiolytic gas production and its effect on plenum operating conditions may require further consideration. During steady-state operation, the system reacts smoothly to reactivity perturbations. Following a significant reactivity step insertion, the system re-establishes equilibrium with a minimum number of power fluctuations. Finally, the system exhibits all the stability characteristics of a very robust system, as evidenced by the open-loop transfer function frequency response. 